\title{
Enhanced recovery after surgery in liver resection: current concepts and controversies
}

\author{
Vandana Agarwal and Jigeeshu V. Divatia \\ Department of Anesthesia, Critical Care and Pain, Tata Memorial Hospital, Homi Bhabha National Institute, \\ Mumbai, Maharashtra, India
}

Enhanced recovery after surgery (ERAS) attenuates the stress response to surgery in the perioperative period and hastens recovery. Liver resection is a complex surgical procedure where the enhanced recovery program has been shown to be safe and effective in terms of postoperative outcomes. ERAS programs have been shown to be associated with lower morbidity, shortened postoperative stay, and reduced cost with no difference in mortality and readmission rates. However, there are challenges that are unique to hepatic resection such as safety after epidural catheterization and postoperative coagulopathy, intraoperative fluids and postoperative organ dysfunction, need for low central venous pressure to reduce blood loss, and non-lactate containing intravenous fluids. This narrative review briefly discusses these concerns and controversies and suggests revisiting some of the strong recommendations made by the ERAS society in light of the recent evidence.

Keywords: Balanced crystalloid; Central venous pressure; Coagulopathy; Enhanced recovery after surgery; Epidural analgesia; Liver resection; Perioperative; Pulse pressure variation; Stroke volume variation; Thromboprophylaxis.

\section{Introduction}

Major abdominal surgery is associated with significant derangement in physiology, including a catabolic state, increased

Corresponding author: Jigeeshu V. Divatia, M.D.

Department of Anesthesia, Critical Care and Pain, Tata Memorial Hospital, Homi Bhabha National Institute, Dr Ernest Borges Marg, Parel, Mumbai 400012, India

Tel: 91-22-24177000, Extension 7041/4042, Fax: 91-22-24146937

Email: jdivatia@yahoo.com

ORCID: https://orcid.org/0000-0001-7384-4886

It was presented at the 95th Annual Scientific Meeting of the Korean Society of Anesthesiologists, November 2018, Seoul Dragon-City Hotelplex, Seoul, Korea.

Received: January 9, 2019.

Revised: March 2, 2019.

Accepted: March 5, 2019.

Korean J Anesthesiol 2019 April 72(2): 119-129

https://doi.org/10.4097/kja.d.19.00010 oxygen demand, impaired pulmonary function, salt and water retention, persistent postoperative pain, nausea and vomiting, delayed recovery of gastrointestinal function, and impaired mobilization with an increased risk of venous thromboembolism, all of which contribute to delayed overall recovery. Enhanced recovery after surgery (ERAS) or fast track surgery was conceptualized by Prof. Henrik Kehlet in the mid-'90s in patients undergoing colorectal surgery and resulted in a significantly shorter postoperative stay [1]. Thereafter the ERAS concept gained considerable momentum globally and results were replicated in other surgical specialties. The ERAS society was formed in the early 2000s and the society has since then published evidence-based guidelines for various surgical procedures (http:// erassociety.org/guidelines/list-of-guidelines/).

Compared to many other abdominal operations, liver resection is a complex surgery with its inherent risks such as prolonged surgery, intraoperative bleeding, associated hypotension and massive fluid shifts, postoperative complications such as coagulopathy, increased risk of bleeding, pulmonary complications, biliary leak, post hepatectomy liver failure, and renal

(c) This is an open-access article distributed under the terms of the Creative Commons Attribution Non-Commercial License (http://creativecommons.org/ licenses/by-nc/4.0/), which permits unrestricted non-commercial use, distribution, and reproduction in any medium, provided the original work is properly cited. 
failure. In addition, these patients may have pre-existing liver disease, presence of cirrhosis, or may have received anti-cancer chemotherapy, which can cause nodular regenerative hyperplasia with non-cirrhotic portal hypertension. These factors make it challenging for both anesthetists and surgeons. Liver resection is associated with a morbidity of $12 \%-46 \%$ and mortality up to $3 \%$ [2]. Fast track concept in liver surgery was reported as early as 2008, long before the published guidelines by the ERAS society for patients undergoing liver resection and was found to be safe and effective with shortened postoperative stay [3]. In this narrative review we briefly discuss the concerns and controversies related to the enhanced recovery concept in patients undergoing liver resections including living donor liver resections. We searched PubMed for papers in English and human subjects evaluating the role of epidural analgesia and other analgesic modalities, coagulation abnormalities, enhanced recovery after surgery and its impact on postoperative outcomes, and hemodynamic management and fluid therapy in the context of liver resection. Papers were searched from 2000 onwards until December 2018. We will focus on issues under the control of the anesthesiologist.

\section{Evidence for ERAS in Liver}

The ERAS society recommends various elements to be incorporated in patients undergoing liver resection. These are listed in Table 1.

Majority of the studies have demonstrated that the ERAS pathway is safe and effective in patients undergoing liver resection and results in shortened postoperative stay [3-5], no difference in mortality, readmission rates [6], and reduced costs [7]. A randomized controlled trial (RCT) in patients undergoing open liver resection compared ERAS with standard care and found that the ERAS group was associated with significantly lower rates of medical complications, shorter stay, and significantly better short term quality of life [8]. Another recent single-center RCT [9] compared ERAS with conventional care and observed early recovery of the synthetic function of the liver, early postoperative gastrointestinal recovery, significantly fewer complications, and lower hospital stay and cost, with no difference in readmissions and re-exploration rates. There was no mortality difference in both the groups. In addition, patient-satisfaction was significantly better in the ERAS group. A systematic review and meta-analysis of seven RCTs including 996 patients demonstrated that the ERAS pathway was associated with significantly lower complications, length of stay, and early recovery of the gastrointestinal function in both laparoscopic and open liver resections. However, six out of the seven RCTs were from China, which may contribute to a certain degree of geographical bias [10]. Liang et al. [11] compared the ERAS pathway with standard care in laparoscopic liver resection and observed that compliance with elements of the ERAS protocol was $86.5 \%$. They found a significant reduction in complications, postoperative stay, cost, and early recovery of gastrointestinal function in ERAS patients with no impact on the conversion and readmission rate.

Table 1. Various Perioperative Elements of ERAS for Liver Resection [12]

\begin{tabular}{|c|c|}
\hline Preoperative elements & $\begin{array}{l}\text { 1. Counseling* } \\
\text { 2. Minimal fasting* } \\
\text { 3. Preoperative carbohydrate load } \\
\text { 4. No oral MBP } \\
\text { 5. Avoid long acting anxiolytics } \\
\text { 6. VTE prophylaxis } \\
\text { 7. Perioperative nutrition }\end{array}$ \\
\hline Intraoperative elements & $\begin{array}{l}\text { 8. Antimicrobial prophylaxis (single dose) and skin preparation ( } 2 \% \text { chlorhexidine })^{*} \\
\text { 9. Perioperative steroids } \\
\text { 10. Maintain normothermia* } \\
\text { 11. Perioperative glycemic control } \\
\text { 12. Abdominal wound catheter/intrathecal opioids instead of epidural analgesia* } \\
\text { 13. Prevention of PONV* } \\
\text { 14. Maintain low CVP during liver resection and use of balanced crystalloids* }\end{array}$ \\
\hline Surgery related & $\begin{array}{l}\text { 15. Avoid Mercedes Benz incision } \\
\text { 16. Minimally invasive surgery where appropriate* } \\
\text { 17. Avoidance of nasogastric tube* } \\
\text { 18. Prevention of DGE by omental flap for left-sided resection }\end{array}$ \\
\hline Postoperative elements & $\begin{array}{l}\text { 19. Early oral intake } \\
\text { 20. Early mobilization } \\
\text { 21. Multimodal analgesia* }\end{array}$ \\
\hline
\end{tabular}

ERAS: enhanced recovery after surgery, MBP: mechanical bowel preparation, VTE: venous thromboembolism, PONV: postoperative nausea and vomiting, CVP: central venous pressure, DGE: delayed gastric emptying. * Implies strong recommendations by the ERAS society. 
The ERAS society published guidelines for liver surgery in 2016 [12] and provided recommendation grades for various elements. However, there are concerns or controversies regarding some of the elements of the ERAS pathway in the context of liver resection. These are related to epidural analgesia and associated postoperative coagulopathy, hypotension and increased infusion of perioperative fluids, postoperative organ dysfunction, venous thromboprophylaxis, fluid strategy (low central venous pressure [CVP] or goal directed fluid therapy), and the type of fluids to be infused.

\section{Concerns with Elements of ERAS}

\section{Epidural analgesia}

Perioperative use of the epidural catheter is one of the key elements in the ERAS pathway for abdominal surgery. Epidural anesthesia and analgesia blunts the perioperative neuroendocrine stress response, provides opioid free postoperative analgesia, and aids in early mobilization and postoperative rehabilitation.

The concerns and controversy with epidural anesthesia in patients undergoing liver surgery are related to the following issues:

A. Postoperative coagulopathy and safety after epidural catheterization.

B. Analgesic efficacy in comparison to other analgesic modalities.

C. Hypotension associated with epidural analgesia, increased perioperative fluid administration, and organ dysfunction.

These will be discussed briefly in light of the current published evidence.

\section{Postoperative coagulopathy and safety after epidural} catheterization

Patients undergoing liver resection have deranged coagulation parameters such as thrombocytopenia, prothrombin time (PT), and international normalized ratio (INR) between postoperative day 1 and 4, and in some cases, this can extend up to 7 days. Elterman and Xiong [13] and Siniscalchi et al. [14] demonstrated a significant decrease in platelet counts and an increase in PT/INR from postoperative day 0 to 5 in patients undergoing major hepatic resection. Postoperative coagulation derangement depends on the pre-existing liver function, preoperative platelet count and INR, volume of liver resection and the future remnant liver volume and its function, intraoperative blood loss more than $1 \mathrm{~L}$, prolonged surgery, presence of cirrhosis, surgical technique, and ischemia reperfusion injury [15]. One of the reasons for the ERAS society's recommendation against epidural analgesia in liver surgery is the presence of postoperative coagulopathy, leading to concerns regarding the safety of removal of the epidural catheter. Postoperative coagulopathy may warrant delaying the removal of the catheter and/or correction of coagulopathy with fresh frozen plasma or platelet transfusion and their associated risks. Also, accidental removal can occur in about $7 \%$ of cases [16]. In a retrospective study of 141 patients, $32 \%$ of patients received vitamin $\mathrm{K}$ or fresh frozen plasma to correct the INR to $<1.3$ before removal of the epidural catheter [13]. However, in patients with liver disease, an elevated INR does not necessarily predict an increased risk of bleeding. In cirrhotics, there is a complex interplay of fluctuating procoagulants and anticoagulants rather than just derangement in INR and low platelets. In patients with chronic liver disease including cirrhosis, there is an increase in factor VIII and von Willebrand factor (vWF), which increases the adhesiveness of platelets to endothelium [17]. Simultaneously there is a decrease in ADAMTS-13, a liver-derived protease that cleaves vWF into smaller and less sticky multimers thus further increasing platelet adhesiveness. In patients with chronic liver disease and cirrhotics, a greater than normal amount of thrombin is generated. In addition, there is a deficiency of protein $\mathrm{C}$, and the ratio of factor VII and protein C is one of the strong determinants of hypercoagulability $[18,19]$. Mallett et al. [20] studied patients with normal liver parenchyma undergoing hepatic resection and observed that the levels of procoagulant factors II, V, VII, and X decreased initially, but the levels of factors $\mathrm{V}$ and $\mathrm{X}$ normalized or exceeded normal range. The levels of factor VIII and vWF also remained elevated with a simultaneous reduction in anticoagulants protein $\mathrm{C}$ and antithrombin by postoperative day 5 . Also, thrombin generation parameters and thromboelastography remained normal throughout the surgical period. This suggests that the hemostatic balance with simultaneous reduction in both pro and anticoagulants and elevated factor VIII and vWF favors a prothrombotic state rather than an increased risk of bleeding, and occurs both in patients with normal liver parenchyma and liver disease with or without cirrhosis.

Siniscalchi et al. [14] did not find any complications related to epidural catheter insertion or removal in cirrhotic patients undergoing liver resection. The catheters were removed between day 3 and 5. Patients with epidural catheters had shorter length of mechanical ventilation and postoperative stay, with no pulmonary or neuraxial complications $[13,14]$.

In the immediate postoperative period after liver resection, PT/INR tests evaluate only the procoagulant activity and not of endogenous anticoagulants and therefore are not appropriate for evaluation of the overall coagulation status in these patients. Viscoelastic testing evaluates both pro- and anticoagulant activity and demonstrates normal, hypercoagulable, or unchanged clot strength after liver resection [20,21]. Hypercoagulability could be one of the reasons why numerous studies evaluating 
the safety of epidural catheters in patients undergoing liver resection have not reported a single epidural or spinal hematoma $[14,22,23]$. However, none of the studies were powered to detect such a rare complication. The American Society of Regional Anesthesia and Pain Medicine (ASRA) [24] guidelines mention avoiding neuraxial blocks in patients with increased PT/INR. However, these recommendations were made for patients on antithrombotics and thrombolytic drugs. There are no specific recommendations for patients with liver disease.

Whilst the balance is restored in patients with liver disease in favor of hypercoagulability, this balance can be easily disturbed by hemodynamic alteration secondary to portal hypertension, bacterial infections, and renal failure; hence an attempt should be made to correct the underlying pathophysiology instead of abnormal coagulation tests. Depending on the underlying pathology these patients can be at an increased risk of bleeding or thrombosis. Conventional tests of coagulation should be interpreted with caution and viscoelastic testing should be used to evaluate the impact on coagulation before the removal of the neuraxial catheter.

\section{Analgesic efficacy}

Epidural analgesia attenuates the stress response to surgery and therefore is incorporated into the ERAS pathway for patients undergoing major abdominal surgery. Compared to general anesthesia alone or postoperative intravenous analgesia, epidural analgesia was associated with the attenuation of surgery-induced stress response and postoperative immunosuppression [25] by maintaining the cytotoxic activity of helper $\mathrm{T}$ cells and natural killer cells in the postoperative period; hence epidural analgesia may be immuno-protective [26]. In addition, it has been shown to be associated with other benefits such as reduced postoperative pulmonary, thromboembolic, and cardiac complications; early mobilization; and recovery of gastrointestinal functions $[14,25,27]$. However, analgesia may be inadequate due to patchy block, accidental dislodgement, pericatheter leak, or early removal because of need for high dependency beds (HDU) beds $[27,28]$.

Epidural analgesia has an opioid-sparing effect within the first $48 \mathrm{~h}$ postoperatively, which may help in early gastrointestinal recovery [29]. Epidural analgesia when functioning well and used appropriately in the postoperative period provides improved analgesia compared to patient-controlled analgesia (PCA) opioids with or without wound catheters and can improve postoperative outcomes [16].

Single shot intrathecal morphine when compared with epidural analgesia provided similar analgesia within the first 48 h. However, patients with intrathecal morphine needed rescue analgesic significantly earlier and had higher incidence of postoperative nausea and pruritus [23]. Koea et al. [4] compared in- trathecal morphine and perioperative Gabapentin with epidural analgesia and demonstrated that intrathecal morphine has similar analgesic effects with significantly decreased fluid demand in the postoperative period, early gastrointestinal recovery, mobilization, and shorter postoperative stay with no difference in complications. Kasivisvanathan et al. [30] compared intrathecal morphine with epidural analgesia and reported similar observations except that epidural analgesia was superior within the first $24 \mathrm{~h}$ after surgery. Although intrathecal morphine provides almost equivalent analgesia and shortened postoperative stay, there is a significantly increased risk of respiratory depression $(3 \%-4 \%)$ within the first $12-16$ h postoperatively [31,32]. Therefore, this mandates that patients should be observed at least in HDU. Also, caution should be exercised in elderly ( $>80$ years), patients with respiratory disease, obese, and obstructive sleep apnea.

Epidural analgesia was found to be superior within the first $48 \mathrm{~h}$ when compared to surgically placed continuous wound infiltration catheters and postoperative PCA opioids, though there was no difference in time to first mobilization [33]. Hughes et al. [34] compared abdominal wound catheters [AWC] $(40 \mathrm{ml}$ of $0.125 \%$ levobupivacaine at the time of insertion of catheters) with epidural analgesia and found epidural analgesia to be significantly superior within $48 \mathrm{~h}$. There was no difference in the levels of inflammatory cytokines postoperatively suggesting similar attenuation with both techniques. Patients in the AWC group had a significantly shorter functional recovery. Epidural analgesia is criticized for a higher failure rate up to $20 \%$ due to inadequate block or inadequate postoperative management. Ganapathi et al. [35] evaluated the safety and efficacy of epidurals in open liver surgery and reported $91 \%$ success rate when managed by a dedicated pain team. Another more recent randomized trial comparing AWC plus PCA versus epidural found epidural to provide superior analgesia following liver resection. Peak flow rate, used as a surrogate for analgesia, was significantly better in the epidural group on POD 1, 2, and 3. Patients with failed epidural received PCA morphine; these patients required significantly more opioids compared to AWC suggesting an opioid-sparing effect of the abdominal fascial catheters and wound infiltration technique [22]. A systematic review evaluating different analgesic modalities on postoperative outcomes after liver surgery found that epidural provided superior analgesia without any effect on postoperative outcomes whereas other analgesic modalities may provide favorable recovery [36]. However, this effect is inconsistent across published studies.

Whilst AWC offers various advantages such as an opioid-sparing effect and early recovery, the success lies in catheter placement and postoperative management. Both intrathecal opioid and AWC catheter need intravenous opioids PCA for maintaining analgesia beyond $48 \mathrm{~h}$. Perioperative use of opioids 
is strongly associated with delayed recovery of gastrointestinal function. In the context of liver resection, this is particularly important as the bioavailability of opioids is increased secondary to decreased drug metabolism and drug accumulation. It also depends on the volume of functioning liver remnant and liver function after surgery. Therefore, when opioids are used they should be used cautiously with appropriate drug, dosage, lockout frequency, and breakthrough doses.

Hypotension associated with epidural analgesia, increased perioperative fluid administration, and organ dysfunction.

Sympathetic blockade and vasodilatation due to thoracic epidural anesthesia may aid the need for deliberate maintenance of low central venous pressure to reduce blood loss during liver resection. Hypotension leads to an increased infusion of fluids intraoperatively and this may continue in the postoperative period. The decrease in mean arterial pressure (MAP) can impair renal perfusion and may lead to acute kidney injury (AKI). Reported incidence of postoperative AKI after liver resection ranges from 8 to $12 \%$ [29,37]. Major liver resection, blood transfusion, pre-existing renal dysfunction, and diabetes are some of the known risk factors for postoperative AKI. In addition, epidural analgesia and associated hypotension in the face of low CVP can augment or increase the risk of AKI [29]. Cirrhotics are a vulnerable group of patients at increased risk of postoperative organ dysfunction secondary to decreased splanchnic perfusion as a result of sympathetic blockade and hypotension following epidural analgesia. Studies have shown that patients with epidural analgesia received an increased infusion of fluids and stayed longer compared to intrathecal opioid [4,30]. Evidence from colonic surgery suggests excessive perioperative fluid administration to be associated with delayed gastrointestinal recovery [38]. In a recent RCT, Bell et al. [22] did not find any difference in the functional gastrointestinal recovery, complications, and stay despite a significantly increased need for fluids in the postoperative period on day 3 in the epidural analgesia group compared to AWC plus intravenous PCA group. Need for vasopressor support was significantly more in epidural analgesia. An increased need for vasopressor support is often used to counteract the sympathetic blockade thus restricting the fluids infused in patients with epidural catheters. Need for vasopressor support mandates invasive blood pressure monitoring and postoperative stay in HDU. This can have an adverse effect on patient mobilization, an integral element in the postoperative component of ERAS pathway. Despite an increased need for invasive monitoring and vasopressor support, there was no difference in HDU stay and time to mobilization between patients with and without epidural catheters [22].

Kambakamba et al. [29] retrospectively studied 829 patients undergoing liver resection and found an overall AKI incidence of $8.2 \%$. Patients receiving epidural analgesia had a significantly higher incidence of AKI (10\% vs. 3.7\%) and were associated with prolonged intensive care unit and hospital stay. However, only $0.8 \%$ patients needed renal replacement therapy. Association of epidural analgesia with AKI was seen mainly in patients undergoing major liver resection. Age, chronic kidney disease, major liver resections, and epidural analgesia were identified as independent predictors of AKI. In contrast, Siniscalchi et al. [14] studied 126 cirrhotic patients with and without epidural anesthesia and found that patients with epidural anesthesia had a significantly lower MAP and required more infusion of colloid; however, there was no difference in the volume of the crystalloids infused and postoperative liver or kidney dysfunction between the groups. On the contrary, patients with epidural analgesia had no respiratory complications and had a significantly shorter postoperative hospital stay. Similarly, when compared to intrathecal morphine, there was no difference in liver and kidney function in the postoperative period despite an increased perioperative fluid infusion in the epidural analgesia group [30].

Hypotension following sympathetic blockade leads to the infusion of an increased volume of fluids, and vasopressors are often used to counteract it, which in turn limits the fluid infusion. Recent studies have demonstrated no difference in the volumes infused and an increased need of vasopressor support with epidural analgesia, thus maintaining the MAP with no difference in postoperative organ dysfunction. It is imperative to note that postoperative organ dysfunction is often multifactorial. Postoperative HDU stay may delay patient mobilization; however, there was no difference in patients with and without epidural analgesia. Alternative modalities such as intrathecal opioids may also mandate HDU stay for respiratory monitoring despite the associated benefits of early functional recovery. A motivated patient and dedicated team within the ERAS program can easily overcome this disadvantage. Epidural seems beneficial in terms of superior analgesia, reduced postoperative respiratory complications, and possibly shorter postoperative stay.

Due to concerns related to postoperative coagulopathy and the safety and efficacy of epidural analgesia, the perceived benefit of epidural analgesia has been questioned in the context of liver resection. Intrathecal morphine, wound infiltration catheters, and abdominal wound/fascial catheters with postoperative intravenous PCA are alternatives to epidural catheters in patients undergoing liver resection. Therefore, the ERAS society recommendation against epidural analgesia in favor of alternative modalities as they provide similar analgesic profile except on day 1 with no difference in postoperative outcomes and recovery [39]. However, recent evidence on coagulation in liver disease suggests a hypercoagulable state in the perioperative period. Whenever possible viscoelastic test should be used for 
evaluating the coagulopathy instead of PT/INR for the decision to remove epidural catheter especially if they are deranged and before correcting coagulopathy. There are conflicting results about the effect of epidural anesthesia on postoperative organ dysfunction. Intrathecal morphine and AWC may be used as an alternative; however, epidural analgesia has been shown to provide superior analgesia on POD 1-3 in addition to an opioid-sparing effect with its associated benefits.

\section{Issue with thromboprophylaxis}

The ERAS society recommends continuation of thromboprophylaxis in the postoperative period until the patient is fully mobile. However, patients may not receive thromboprophylaxis until the PT/INR normalizes in the postoperative period. In a recent survey, $35 \%$ of the centers did not administer thromboprophylaxis in the postoperative period until the INR was normal [40]. Most studies evaluating liver resections have reported an incidence of venous thromboembolism (VTE) ranging from $2.8 \%$ to $4.7 \%$ [41-43]. Tzeng et al. [43] evaluated 5,651 open hepatectomies using the National Surgical Quality Improvement Program (NSQIP) database and observed an incidence of $2.8 \%$ for VTE and $1.3 \%$ for pulmonary embolism. The incidence of VTE increased with the extent of hepatic resection from $2.1 \%$ to $5.8 \%$. Patients with VTE also had a significantly higher mortality (7.4\% vs. $2.3 \%)$. A systematic review evaluated the safety and efficacy of pharmacological prophylaxis following liver resection and found that in a pooled cohort of 3,675 patients, $61 \%$ patients received pharmacological prophylaxis. They found a significantly lower incidence of VTE in patients who received pharmacological prophylaxis compared to those who did not (2.6\% vs. $4.6 \%, \mathrm{P}=0.030$, respectively) [44]. Above studies reveal that VTE is a serious complication following liver resection despite deranged PT/INR values.

Thus the recommendation for perioperative venous thromboprophylaxis needs to be further emphasized as the balance between pro- and anticoagulants in patients undergoing liver resection may predispose them to an increased risk of thrombosis. Viscoelastic test may be considered to assess the effect on coagulation and prescription of pharmacological prophylaxis, particularly when PT/INR are deranged.

\section{Fluid management - low CVP vs. other modalities of fluid responsiveness and type of fluid}

a) Low CVP of 2-5 $\mathrm{mmHg}$, total hepatic inflow occlusion during liver resection, and acute normovolemic hemodilution (if estimated blood loss $>20 \%$ ) are associated with reduced blood loss and transfusion rates and lower morbidity and mortality $[45,46]$. Low CVP reduces the distension of the central veins and thus aids in dissection. Further during parenchymal dissection venous bleeding from the hepatic veins is reduced because of reduced outflow pressure. This is often achieved by patient position (head up tilt), acute normovolemic intraoperative hemodilution, restriction of fluids, use of thoracic epidural anesthesia, and use of vasodilators such as nitroglycerin, milrinone, or diuretics [47]. The optimum technique of lowering CVP is not known. In a systematic review and meta-analysis of randomized control trials, low CVP was associated with a significant reduction in blood loss and transfusion rates. The anesthetic measures used to reduce CVP significantly reduced blood loss compared to only surgical maneuvers. However, there was no difference in the morbidity or postoperative stay between the surgical and anesthetic techniques of lowering CVP [48]. However, on the other hand, there is also evidence that low CVP is not associated with reduced blood loss in living donor hepatectomy $[49,50]$. Whilst reduction in CVP decreases blood loss, CVP can be affected by tricuspid valve disease, pulmonary disease, and intra-pericardial and intra-abdominal pressures. CVP can be elevated due to mechanical factors such as surgical retractors on the diaphragm; compression of the liver during surgery can release a significant amount of blood. Similarly, it can be lower due to mechanical compression of the portal vein and inferior vena cava during dissection or clamping of the porta. Also, placement of transducer can have a huge impact on the measured value and its subsequent interpretation. Evidence in intensive care suggests CVP should not be used to assess the fluid volume status [51-53]. In a questionnaire survey among the anesthesiologists and intensivists to identify the best place for alignment with the phlebostatic axis for CVP measurement, only $3.4 \%$ identified correctly and $40 \%$ of the respondents mentioned CVP had no clear cut relation to volume status [54]. Low CVP, on the contrary, can increase the risk of inadvertent organ hypoperfusion in the event of bleeding and air embolism.

Correa-Gallego et al. [55] evaluated the effect of low CVP on renal function. They retrospectively analyzed a prospectively maintained database for postoperative incidence of biochemical and clinically relevant AKI using the Modification of Diet in Renal Disease for estimating the glomerular filtration rate. They found that $17 \%$ patients had biochemical AKI and only $1 \%$ experienced failure, which resolved in half of these patients after a short follow-up period. Sand et al. [56] advise against head up position as a $10^{\circ}$ head up tilt with $5 \mathrm{cmH}_{2} \mathrm{O}$ positive end expiratory pressure (PEEP) decreased the CVP and MAP with no difference in the hepatic and portal venous pressure.

b) Stroke volume variation (SVV) is one of the dynamic indices of hemodynamic monitoring in mechanically ventilated patients to assess fluid responsiveness, i.e. a significant increase in stroke volume or cardiac output from the baseline after administration of a bolus of fluid. Choi et al. [57] investigated the 
impact of low (<10\%) vs. high SVV $(10 \%-20 \%)$ on intraoperative blood loss and postoperative outcomes, and as an alternative to CVP monitoring in patients undergoing living donor right hepatectomy. They found that high SVV was associated with significantly reduced blood loss with no difference in postoperative organ dysfunction, morbidity, mortality, and stay. Incidentally, the high SVV group also had significantly lower CVP (median $2.7 \mathrm{mmHg}$ ) during hepatectomy; hence the authors could not disentangle the effect of low CVP on blood loss. Dunki-Jacobs et al. [58] evaluated the correlation between SVV and CVP and also the safety and efficacy of SVV as an alternative to CVP in patients undergoing open and laparoscopic hepatectomy. They found that CVP $<3 \mathrm{mmHg}$ corresponded to SVV $>$ $13 \%$, and CVP of -1 to $1 \mathrm{mmHg}$ significantly correlated to SVV of $18 \%-21 \%$. Similar trend was seen in patients with open and laparoscopic hepatectomy. Therefore, SVV has an advantage in patients undergoing laparoscopic resections where CVP loses its value. Kitaguchi et al. [59] used SVV > 13\%-20\% and fluid restriction $<5 \mathrm{ml} / \mathrm{kg} / \mathrm{h}$ during hepatic resection compared to a historical cohort and found a significant decrease in blood loss and transfusion rates. Ratti et al. [60] conducted a randomized trial comparing fluid management guided by SVV or CVP in patients undergoing laparoscopic left lateral resection. They observed that the blood loss was significantly lower in the SVV group. The CVP group had a higher conversion rate because of hemorrhage and consequentially increased blood loss.

c) Pulse pressure variation (PPV) is another dynamic index of fluid responsiveness derived from the arterial waveform. Ferrario et al. [61] evaluated various dynamic indices in liver surgery using different methods of cardiac output estimation namely systolic area (SA) and the Liljestrand and Zander (LM) method. They observed that PPV was more reliable than SVV. PPV provided by PiCCO (Pulsion Medical Systems SE, Germany) performed best in terms of area under curve, sensitivity, and specificity $(0.92,0.88$, and 0.86 , respectively). SVV showed good performance; however, there were differences between the estimators (PiCCO, SA, and LM). The PPV threshold value (14\%) was similar to published literature; however, the threshold value of SVV ranged from 9.4 to 19.7 depending on the method of estimation. SVV derived from PiCCO and SA methods were very similar - 18.2 and 19.7, respectively. Dunki-Jacobs et al. [58] observed similar value in their study.

In light of recent evidence, SVV correlates well with CVP in both open and laparoscopic hepatectomies, and requires an arterial line, which has very low incidence of complications $(<1 \%)$. CVP, on the hand, is limited by the various factors mentioned above and requires central venous cannulation with complication ranging from $5 \%$ to $15 \%$ [57]. Similarly, PPV can also be used as a measure of fluid responsiveness with a threshold value of $14 \%$; however, further studies are needed to validate it in a larger number of patients undergoing liver resection. PPV has an additional advantage; it can be derived from an arterial waveform through an algorithm built in the monitor and does not need a cardiac output monitor, unlike SVV. These dynamic indices have certain limitations. They are unreliable in patients with arrhythmias and during mechanical ventilation with a tidal volume of $<8 \mathrm{ml} / \mathrm{kg}$ of predicted body weight. Risk-benefit ratio of the above mentioned hemodynamic indices favors SVV and PPV. Central venous cannulation should be considered in patients with difficult vascular access or need for parenteral nutrition postoperatively. The ERAS society's strong recommendation for the maintenance of low CVP during hepatic resection should be re-evaluated in view of the limitations of CVP and the evidence favoring the dynamic indices of fluid responsiveness.

d) Type of fluids. The main concern in patients undergoing liver resection is postoperative renal dysfunction and lactate clearance. Preoperative liver or renal disease, intraoperative hemodynamic and fluid management, surgical handling, blood loss, replacement fluid, and impaired remnant liver function can cause postoperative renal dysfunction. Lactate clearance can be affected by the volume and function of remnant liver, clinically overt/covert hypovolemia, and possibly due to the administration of lactate containing fluids. Wiggans et al. [62] found initial (first) postoperative lactate concentration to be associated with an increased risk of liver and renal dysfunction and mortality. Lactate $>6 \mathrm{mmol} / \mathrm{L}$ was associated with significant renal dysfunction and 90 -day mortality ( $28 \%$ vs. $0.7 \%)$. The presence of diabetes, surgical assessment of the liver at laparotomy, the extent of liver resection, blood loss, and the number of units of blood transfused were associated with post-operative serum lactate concentration. Since the liver is the primary organ to metabolize lactate there's concern regarding the ability of the liver to convert lactate to bicarbonate in the presence of hepatic insufficiency during and after hepatectomy. Acetate, on the other hand, is metabolized by various other organ systems. Therefore, the choice of the fluid (lactate or acetate containing) administered in these patients is important.

The ERAS society strongly recommends the use of balanced crystalloid and to avoid $0.9 \%$ normal saline. McCluksey et al. [63] conducted a retrospective cohort trial and observed a $22 \%$ incidence of postoperative hyperchloremia in non-transplant, non-cardiac surgery. Hyperchloremia was associated with postoperative renal dysfunction, increased mortality, and longer postoperative stay. Another large database study found the intraoperative administration of saline compared to balanced crystalloid to be associated with significantly more major complications (postoperative infection, renal failure requiring dialysis, blood transfusion, acidosis, and electrolyte derangements), mortality, and resource utilization in patients undergoing open major abdominal surgery [64]. Evidence from studies in the 
emergency department [65] and intensive care [66] favors balanced crystalloids in favor of normal saline. Balanced crystalloid was associated with improved renal outcomes. However, there is no information on how many patients had undergone liver surgery. Kumar et al. [67] evaluated the metabolic effect of the combination of Ringer's lactate before resection followed by normal saline during resection with acetate-based balanced crystalloid in living donor hepatectomy. They found no difference in the lactate levels between the groups, but bicarbonate was lower; base deficit and chloride was increased in the control group probably secondary to normal saline.

Shin et al. [68] conducted a single-center RCT comparing acid base status, lactate concentrations, and liver function test in patients who received Plasmalyte ${ }^{\mathrm{TM}}-148$ (PL) or Hartmann's solution (HS) in 104 donors undergoing right hepatectomy. PL resulted in lower lactate and bilirubin levels, lower PT, and higher albumin levels compared to patients receiving Hartmann's solution. However, there were no significant differences in complications or duration of hospital stay between the groups. Weinberg et al. [69] conducted a multicenter, double-blind RCT to evaluate biochemical and hematological effects of HS with PL in patients undergoing major hepatic resection. Patients in the HS group had hyperchloremia and hyperlactatemia (77\% vs. $47 \%$ ). Patients in the PL group had higher magnesium and lower ionized calcium and an effective strong ion difference after surgery. There was no difference in $\mathrm{pH}$, bicarbonate, albumin, and phosphate levels. The authors also observed a significantly lower rate of complications and stay in the PL group. The authors suggest that the effect on clinical outcomes be interpreted with caution as the study was powered to detect biochemical changes (primary end-point) and not clinical outcomes. The effect on clinical outcomes could be a chance finding.

The strong recommendation by the ERAS society is based on the studies by McCluskey et al. [63] in patients undergoing non-cardiac, non-transplant surgery, and Shaw et al. [64]. In these studies there is no information about how many patients underwent liver surgery. Lactated solutions may cause hyperlactatemia and acidosis and other biochemical changes in patients undergoing liver surgery, but none of the studies so far have demonstrated the benefit of balanced crystalloids on the important clinical endpoints such as morbidity, mortality, and stay. Future trials may shed light on this issue.

\section{Summary}

In light of new data, the controversies and concerns with some of the ERAS elements formulated for liver surgery in 2016 seem valid. These revolve around the role of thoracic epidural catheterization, associated hypotension and postoperative organ dysfunction, thromboprophylaxis, need for low CVP, and type of fluids to be administered. The recommendation against the use of thoracic epidural catheterization in favor of AWC is driven by concerns regarding the safety of the removal of epidural catheters in the presence of postoperative coagulopathy. Recent data suggest that the PT/INR are inadequate tests to evaluate the coagulopathy in patients undergoing liver surgery. There is a rebalance of procoagulants and anticoagulants that may favor a prothrombotic state after liver resection. The association of postoperative liver and kidney dysfunction with intraoperative hypotension as sequelae of epidural analgesia with need for an increased infusion of fluids is inconsistent in the published literature. It is important to note that the occurrence of postoperative AKI and liver dysfunction is dependent on a multitude of factors. Despite increased intraoperative fluids, there was no difference in the recovery of bowel function. Epidural analgesia has an opioid-sparing effect with a failure rate similar to alternative modalities when managed postoperatively by a dedicated pain team. It provides superior analgesia within the first $72 \mathrm{~h}$ compared to AWCs. Whilst AWC and intrathecal morphine with intravenous PCA opioid have a more favorable recovery profile, catheter placement is crucial for a successful block and the risk of respiratory depression mandating HDU stay is always a concern with intrathecal morphine. These alternative modalities are often used in conjunction with intravenous PCA opioid, which may be associated with nausea and pruritus. Therefore, considering the benefits versus harm in light of recent evidence, epidural analgesia seems safe in liver resection. The ERAS society does recommend continuation of venous thromboprophylaxis postoperatively until the patient is fully mobile. However, the guideline makes no specific mention about postoperative thromboprophylaxis in the presence of postoperative coagulopathy. Venous thromboprophylaxis should be administered to these patients. If PT/INR is suggestive of coagulopathy, viscoelastic tests should be done, and thromboprophylaxis should be administered unless viscoelastic coagulation tests are suggestive of a bleeding coagulopathy. Viscoelastic tests are more appropriate in these patients to evaluate the postoperative coagulation status and to guide the correction of any coagulopathy. The ERAS society recommends the maintenance of low CVP with close monitoring during hepatic resection and the use of balanced crystalloids over normal saline to maintain intravascular volume, avoid hyperchloremic acidosis, and renal dysfunction. However, recent evidence suggests dynamic indices of fluid responsiveness could replace CVP. Central venous cannulation will have its place in patients with difficult venous access and the need for postoperative parenteral nutrition. Currently, there is no evidence for using balanced crystalloids in terms of improved postoperative outcomes in patients undergoing liver resection. The ERAS society guidelines for liver surgery were published in 2016; however, recent evidence suggests revisiting some of the 
recommendations. Future research will guide the role of alternative modalities in comparison to epidural analgesia and hemodynamic targets, indices, and type of fluids to be used in patients undergoing liver resection.

\section{Conflicts of Interest}

No potential conflict of interest relevant to this article was reported.

\section{Author Contributions}

Vandana Agarwal (Methodology; Writing - original draft)

Jigeeshu V. Divatia (Conceptualization; Methodology; Supervision; Writing - review \& editing)

\section{ORCID}

Vandana Agarwal, https://orcid.org/0000-0002-3593-0414

Jigeeshu V. Divatia, https://orcid.org/0000-0001-7384-4886

\section{References}

1. Basse L, Hjort Jakobsen D, Billesbølle P, Werner M, Kehlet H. A clinical pathway to accelerate recovery after colonic resection. Ann Surg 2000; 232: 51-7.

2. Damania R, Cocieru A. Impact of enhanced recovery after surgery protocols on postoperative morbidity and mortality in patients undergoing routine hepatectomy: review of the current evidence. Ann Transl Med 2017; 5: 341.

3. van Dam RM, Hendry PO, Coolsen MM, Bemelmans MH, Lassen K, Revhaug A, et al. Initial experience with a multimodal enhanced recovery programme in patients undergoing liver resection. Br J Surg 2008; 95: 969-75.

4. Koea JB, Young Y, Gunn K. Fast track liver resection: the effect of a comprehensive care package and analgesia with single dose intrathecal morphine with gabapentin or continuous epidural analgesia. HPB Surg 2009; 2009: 271986.

5. Savikko J, Ilmakunnas M, Mäkisalo H, Nordin A, Isoniemi H. Enhanced recovery protocol after liver resection. Br J Surg 2015; 102: 152632 .

6. Rouxel P, Beloeil H. Enhanced recovery after hepatectomy: a systematic review. Anaesth Crit Care Pain Med 2019; 38: 29-34.

7. Joliat GR, Labgaa I, Hübner M, Blanc C, Griesser AC, Schäfer M, et al. Cost-benefit analysis of the implementation of an enhanced recovery program in liver surgery. World J Surg 2016; 40: 2441-50.

8. Jones C, Kelliher L, Dickinson M, Riga A, Worthington T, Scott MJ, et al. Randomized clinical trial on enhanced recovery versus standard care following open liver resection. Br J Surg 2013; 100: 1015-24.

9. Qi S, Chen G, Cao P, Hu J, He G, Luo J, et al. Safety and efficacy of enhanced recovery after surgery (ERAS) programs in patients undergoing hepatectomy: a prospective randomized controlled trial. J Clin Lab Anal 2018: e22434.

10. Zhao Y, Qin H, Wu Y, Xiang B. Enhanced recovery after surgery program reduces length of hospital stay and complications in liver resection: a PRISMA-compliant systematic review and meta-analysis of randomized controlled trials. Medicine (Baltimore) 2017 ; 96 : e7628.

11. Liang X, Ying H, Wang H, Xu H, Liu M, Zhou H, et al. Enhanced recovery care versus traditional care after laparoscopic liver resections: a randomized controlled trial. Surg Endosc 2018; 32: 2746-57.

12. Melloul E, Hübner M, Scott M, Snowden C, Prentis J, Dejong CH, et al. Guidelines for Perioperative Care for Liver Surgery: Enhanced Recovery After Surgery (ERAS) Society Recommendations. World J Surg 2016; 40: 2425-40.

13. Elterman KG, Xiong Z. Coagulation profile changes and safety of epidural analgesia after hepatectomy: a retrospective study. J Anesth 2015; 29: 367-72.

14. Siniscalchi A, Gamberini L, Bardi T, Laici C, Gamberini E, Francorsi L, et al. Role of epidural anesthesia in a fast track liver resection protocol for cirrhotic patients - results after three years of practice. World J Hepatol 2016; 8: 1097-104.

15. Jacquenod P, Wallon G, Gazon M, Darnis B, Pradat P, Virlogeux V, et al. Incidence and risk factors of coagulation profile derangement after liversurgery: implications for the use of epidural analgesia-a retrospectivecohort study. Anesth Analg 2018; 126: 1142-7.

16. Tzimas P, Prout J, Papadopoulos G, Mallett SV. Epidural anaesthesia and analgesia for liver resection. Anaesthesia 2013; 68: 628-35.

17. Tripodi A, Mannucci PM. The coagulopathy of chronic liver disease. N Engl J Med 2011; 365: 147-56.

18. Tripodi A. Hemostasis abnormalities in cirrhosis. Curr Opin Hematol 2015; 22: 406-12.

19. Stravitz RT. Potential applications of thromboelastography in patients with acute and chronic liver disease. Gastroenterol Hepatol (N Y) 2012; 8: 513-20.

20. Mallett SV, Sugavanam A, Krzanicki DA, Patel S, Broomhead RH, Davidson BR, et al. Alterations in coagulation following major liver resection. Anaesthesia 2016; 71: 657-68.

21. Barton JS, Riha GM, Differding JA, Underwood SJ, Curren JL, Sheppard BC, et al. Coagulopathy after a liver resection: is it over diagnosed and over treated? HPB (Oxford) 2013; 15: 865-71.

22. Bell R, Ward D, Jeffery J, Toogood GJ, Lodge JA, Rao K, et al. A randomized controlled trial comparing epidural analgesia versus continuous local anesthetic infiltration via abdominal wound catheter in open liver resection. Ann Surg 2019; 269: 413-9. 
23. De Pietri L, Siniscalchi A, Reggiani A, Masetti M, Begliomini B, Gazzi M, et al. The use of intrathecal morphine for postoperative pain relief after liver resection: a comparison with epidural analgesia. Anesth Analg 2006; 102: 1157-63.

24. Horlocker TT, Vandermeuelen E, Kopp SL, Gogarten W, Leffert LR, Benzon HT. Regional anesthesia in the patient receiving antithrombotic or thrombolytic therapy: American Society of Regional Anesthesia and Pain Medicine Evidence-Based Guidelines (Fourth Edition). Reg Anesth Pain Med 2018; 43: 263-309.

25. Nimmo SM. Benefit and outcome after epidural analgesia. Contin Educ Anaesth Crit Care Pain 2004; 4: 44-7.

26. Gu CY, Zhang J, Qian YN, Tang QF. Effects of epidural anesthesia and postoperative epidural analgesia on immune function in esophageal carcinoma patients undergoing thoracic surgery. Mol Clin Oncol 2015; 3: 190-6.

27. Rigg JR, Jamrozik K, Myles PS, Silbert BS, Peyton PJ, Parsons RW, et al. Epidural anaesthesia and analgesia and outcome of major surgery: a randomised trial. Lancet 2002; 359: 1276-82.

28. Revie EJ, Massie LJ, McNally SJ, McKeown DW, Garden OJ, Wigmore SJ. Effectiveness of epidural analgesia following open liver resection. HPB (Oxford) 2011; 13: 206-11.

29. Kambakamba P, Slankamenac K, Tschuor C, Kron P, Wirsching A, Maurer K, et al. Epidural analgesia and perioperative kidney function after major liver resection. Br J Surg 2015; 102: 805-12.

30. Kasivisvanathan R, Abbassi-Ghadi N, Prout J, Clevenger B, Fusai GK, Mallett SV. A prospective cohort study of intrathecal versus epidural analgesia for patients undergoing hepatic resection. HPB (Oxford) 2014; 16: 768-75.

31. Dichtwald S, Ben-Haim M, Papismedov L, Hazan S, Cattan A, Matot I. Intrathecal morphine versus intravenous opioid administration to impact postoperative analgesia in hepato-pancreatic surgery: a randomized controlled trial. J Anesth 2017; 31: 237-45.

32. Sakowska M, Docherty E, Linscott D, Connor S. A change in practice from epidural to intrathecal morphine analgesia for hepatopancreato-biliary surgery. World J Surg 2009; 33: 1802-8.

33. Revie EJ, McKeown DW, Wilson JA, Garden OJ, Wigmore SJ. Randomized clinical trial of local infiltration plus patient-controlled opiate analgesia vs. epidural analgesia following liver resection surgery. HPB (Oxford) 2012; 14: 611-8.

34. Hughes MJ, Harrison EM, Peel NJ, Stutchfield B, McNally S, Beattie C, et al. Randomized clinical trial of perioperative nerve block and continuous local anaesthetic infiltration via wound catheter versus epidural analgesia in open liver resection (LIVER 2 trial). Br J Surg 2015; 102: 1619-28.

35. Ganapathi S, Roberts G, Mogford S, Bahlmann B, Ateleanu B, Kumar N. Epidural analgesia provides effective pain relief in patients undergoing open liver surgery. Br J Pain 2015; 9: 78-85.

36. Hughes M, McNally S, McKeown DW, Wigmore S. Effect of analgesic modality on outcome following open liver surgery: a systematic review of postoperative analgesia. Minerva Anestesiol 2015; 81: 541-56.

37. Tomozawa A, Ishikawa S, Shiota N, Cholvisudhi P, Makita K. Perioperative risk factors for acute kidney injury after liver resection surgery: an historical cohort study. Can J Anaesth 2015; 62: 753-61.

38. Lobo DN, Bostock KA, Neal KR, Perkins AC, Rowlands BJ, Allison SP. Effect of salt and water balance on recovery of gastrointestinal function after elective colonic resection: a randomised controlled trial. Lancet 2002; 359: 1812-8.

39. Bell R, Pandanaboyana S, Prasad KR. Epidural versus local anaesthetic infiltration via wound catheters in open liver resection: a metaanalysis. ANZ J Surg 2015; 85: 16-21.

40. Weiss MJ, Kim Y, Ejaz A, Spolverato G, Haut ER, Hirose K, et al. Venous thromboembolic prophylaxis after a hepatic resection: patterns of care among liver surgeons. HPB (Oxford) 2014; 16: 892-8.

41. Ejaz A, Spolverato G, Kim Y, Lucas DL, Lau B, Weiss M, et al. Defining incidence and risk factors of venous thromboemolism after hepatectomy. J Gastrointest Surg 2014; 18: 1116-24.

42. Turley RS, Reddy SK, Shortell CK, Clary BM, Scarborough JE. Venous thromboembolism after hepatic resection: analysis of 5,706 patients. J Gastrointest Surg 2012; 16: 1705-14.

43. Tzeng CW, Katz MH, Fleming JB, Pisters PW, Lee JE, Abdalla EK, et al. Risk of venous thromboembolism outweighs post-hepatectomy bleeding complications: analysis of 5651 National Surgical Quality Improvement Program patients. HPB (Oxford) 2012; 14: 506-13.

44. Baltatzis M, Low R, Stathakis P, Sheen AJ, Siriwardena AK, Jamdar S. Efficacy and safety of pharmacological venous thromboembolism prophylaxis following liver resection: a systematic review and meta-analysis. HPB (Oxford) 2017; 19: 289-96.

45. Huntington JT, Royall NA, Schmidt CR. Minimizing blood loss during hepatectomy: a literature review. J Surg Oncol 2014; $109: 81-8$.

46. Wang WD, Liang LJ, Huang XQ, Yin XY. Low central venous pressure reduces blood loss in hepatectomy. World J Gastroenterol 2006; 12: 935-9.

47. Page AJ, Kooby DA. Perioperative management of hepatic resection. J Gastrointest Oncol 2012; 3: 19-27.

48. Hughes MJ, Ventham NT, Harrison EM, Wigmore SJ. Central venous pressure and liver resection: a systematic review and meta-analysis. HPB (Oxford) 2015; 17: 863-71.

49. Kim YK, Chin JH, Kang SJ, Jun IG, Song JG, Jeong SM, et al. Association between central venous pressure and blood loss during hepatic resection in 984 living donors. Acta Anaesthesiol Scand 2009; 53: 601-6.

50. Niemann CU, Feiner J, Behrends M, Eilers H, Ascher NL, Roberts JP. Central venous pressure monitoring during living right donor hepatectomy. Liver Transpl 2007; 13: 266-71.

51. Marik PE, Baram M, Vahid B. Does central venous pressure predict fluid responsiveness? A systematic review of the literature and the tale 
of seven mares. Chest 2008; 134: 172-8.

52. Marik PE, Cavallazzi R. Does the central venous pressure predict fluid responsiveness? An updated meta-analysis and a plea for some common sense. Crit Care Med 2013; 41: 1774-81.

53. Polderman KH, Varon J, Marik PE. Fluid management decisions should not be guided by fixed central venous pressure targets. Am J Emerg Med 2015; 33: 1311.

54. Sondergaard S, Parkin G, Aneman A. Central venous pressure: we need to bring clinical use into physiological context. Acta Anaesthesiol Scand 2015; 59: 552-60.

55. Correa-Gallego C, Berman A, Denis SC, Langdon-Embry L, O'Connor D, Arslan-Carlon V, et al. Renal function after low central venous pressure-assisted liver resection: assessment of 2116 cases. HPB (Oxford) 2015; 17: 258-64.

56. Sand L, Rizell M, Houltz E, Karlsen K, Wiklund J, Odenstedt Hergès H, et al. Effect of patient position and PEEP on hepatic, portal and central venous pressures during liver resection. Acta Anaesthesiol Scand 2011; 55: 1106-12.

57. Choi SS, Kim SH, Kim YK. Fluid management in living donor hepatectomy: Recent issues and perspectives. World J Gastroenterol 2015; 21: 12757-66.

58. Dunki-Jacobs EM, Philips P, Scoggins CR, McMasters KM, Martin RC 2nd. Stroke volume variation in hepatic resection: a replacement for standard central venous pressure monitoring. Ann Surg Oncol 2014; 21: 473-8.

59. Kitaguchi K, Gotohda N, Yamamoto H, Takahashi S, Konishi M, Hayashi R. A comparative study of intraoperative fluid management using stroke volume variation in liver resection. Int Surg 2017. Advance Access published on Nov 15, 2017. doi:10.9738/INTSURG-D-17-00094.1

60. Ratti F, Cipriani F, Reineke R, Catena M, Paganelli M, Comotti L, et al. Intraoperative monitoring of stroke volume variation versus central venous pressure in laparoscopic liver surgery: a randomized prospective comparative trial. HPB (Oxford) 2016; 18: 136-44.

61. Ferrario M, Pala S, Aletti F, Toschi N, Canichella A, Guerrisi M, et al. Fluid responsiveness in liver surgery: comparisons of different indices and approaches. J Comput Surg 2014; $1: 6$.

62. Wiggans MG, Starkie T, Shahtahmassebi G, Woolley T, Birt D, Erasmus P, et al. Serum arterial lactate concentration predicts mortality and organ dysfunction following liver resection. Perioper Med (Lond) 2013; 2: 21.

63. McCluskey SA, Karkouti K, Wijeysundera D, Minkovich L, Tait G, Beattie WS. Hyperchloremia after noncardiac surgery is independently associated with increased morbidity and mortality: a propensity-matched cohort study. Anesth Analg 2013; 117: 412-21.

64. Shaw AD, Bagshaw SM, Goldstein SL, Scherer LA, Duan M, Schermer CR, et al. Major complications, mortality, and resource utilization after open abdominal surgery: 0.9\% saline compared to Plasma-Lyte. Ann Surg 2012; 255: 821-9.

65. Self WH, Semler MW, Wanderer JP, Wang L, Byrne DW, Collins SP, et al. Balanced crystalloids versus saline in noncritically Ill adults. N Engl J Med 2018; 378: 819-28.

66. Semler MW, Self WH, Wanderer JP, Ehrenfeld JM, Wang L, Byrne DW, et al. Balanced crystalloids versus saline in critically Ill adults. N Engl J Med 2018; 378: 829-39.

67. Kumar L, Seetharaman M, Rajmohan N, Ramamurthi P, Rajan S, Varghese R. Metabolic profile in right lobe living donor hepatectomy: comparison of lactated Ringer's solution and normal saline versus acetate based balanced salt solution - a pilot study. Indian J Anaesth 2016; 60: 719-25.

68. Shin WJ, Kim YK, Bang JY, Cho SK, Han SM, Hwang GS. Lactate and liver function tests after living donor right hepatectomy: a comparison of solutions with and without lactate. Acta Anaesthesiol Scand 2011; 55: 558-64.

69. Weinberg L, Pearce B, Sullivan R, Siu L, Scurrah N, Tan C, et al. The effects of plasmalyte-148 vs. Hartmann's solution during major liver resection: a multicentre, double-blind, randomized controlled trial. Minerva Anestesiol 2015; 81: 1288-97. 